\title{
Cancer
}

\section{Repositioned to kill stem cells}

Chemotherapy-resistant cancer stem cells preclude cure to many forms of the disease. Repositioning an existing drug to tackle this problem could significantly improve treatment for one form of leukaemia. See Letter p.XXX

\section{Tessa Holyoake \& David Vetrie}

In most cases of chronic myeloid leukaemia (CM L), a daily oral medication rapidly transforms a progressive and ultimately fatal cancer into a chronic, but manageable condition. But this is not a cure. The persistence of quiescent (dormant, non-cycling) and thus drugresistant leukaemic stem cells (LSCs) represents an unmet clinical challenge in CM L, and any attempt at cure must specifically target the eradication of these cells. In a paper published on Nature's website today, Prost et al. ${ }^{1}$ present provocative pre-clinical and early clinical findings demonstrating that a drug currently used for diabetes therapy can be repositioned to target a pathway controlling quiescence in LSCs, causing the gradual erosion of this cellular pool.

CM L arises in a normal blood stem cell as a result of a mutation that involves an exchange of genetic material between chromosomes 9 and 22. This translocation creates the cancerdriving gene known as $B C R-A B L 1$, which produces a protein with enhanced activity as a tyrosine kinase enzyme, leading to uncontrolled cell proliferation. BCR-A BL 1 has been shown to be sufficient to drive the development of leukaemia in mouse models ${ }^{2}$, and the 
discovery of this protein led to the development of tyrosine kinase inhibitors (TK Is) for CM L treatment.

Over the past two decades, TK Is have dramatically improved the outcome for patients with this cancer. M ost patients presenting with early disease respond rapidly to TKI therapy and go into long-lasting remission. However, TK Is fail to eradicate LSCs, the cells that initiate and maintain $C M L$, and these drug-resistant cells can drive relapse, or evolve to cause further forms of TK I resistance and more aggressive disease. As a result, patients on life-long TK I therapy are exposed to associated, often serious, side effects and may cease to respond to the treatment at any time. Furthermore, the significantly improved survival for patients on TKIS means that disease prevalence is increasing each year, with inherent social and economic implications.

Several potential mechanisms to explain the insensitivity of LSCs to TK Is have been proposed, among which cellular quiescence seems to be key. Prost et al. describe that quiescence in LSCs is regulated by a pathway involving the receptor PPA ${ }^{3}$, the transcription factors STA T5 and HIF $2 \alpha$, and the protein CITED2, known as a master regulator of blood stem cell quiescence (Fig. 1). A particular strength of the study was the use of primary blood stem cells (expressing the marker CD34) from patients with CM L to dissect the pathway and confirm the role of each component in regulating LSC quiescence.

The authors go on to show that combining imatinib, the standard TKI used to manage CM L, with the antidiabetic agent pioglitazone, which activates PPA ${ }^{3}$, blocks this pathway in CM L cells. The synergistic effects of the drugs reduce STA T 5 expression and activity, downregulate HIF $2 \pm$ and CITED2 expression, and trigger the death of quiescent LSCS. 
A lthough the mechanism by which LSCs are killed in response to this drug combination is not clear, it is probable that they are either directly killed or driven to exit quiescence, which may predispose them to being eradicated by the TKI. The authors also demonstrate that the compound J Q1, a bromodomain inhibitor with broad activity that includes suppression of STAT5 activity, is as effective as pioglitazone (in combination with imatinib). A lthough this finding supports the role for the STAT5 pathway in LSC quiescence, the door is still open for studies of other agents that may target LSCs through this or alternative pathways.

Collectively, these results strengthen the concept that cancer stem cells exhibit vulnerabilities in otherwise normal molecular pathways that may be targeted in a selective manner to achieve cure. Earlier work has demonstrated that CM L stem cell quiescence is in part maintained by the promyelocytic leukaemia tumour-suppressor protein, which can be targeted by arsenic trioxide ${ }^{3}$, and that the cellular catabolic process of autophagy functions as a survival pathway for CML stem cells that can be targeted by the repositioning of the antimalarial agent hydroxychloroquine ${ }^{4}$ (Fig. 1). B oth of these approaches are currently under investigation in the clinic.

Prost el al. also tested the addition of pioglitazone to imatinib therapy in three patients with $C M L$, and found that the patients converted from having demonstrable residual leukaemia to being disease-free, and that this effect lasted for months to years after pioglitazone withdrawal. These data provided a strong rationale for a phase 2 clinical trial, which started in J uly 2009 (ACTIM EudraCT 2009-011675-79). A though the interim results from this trial are encouraging, the study is non-randomised and it will therefore be difficult to ascertain definitively that improved response rates are driven by pioglitazone. 
Despite the need for further clinical testing of this particular combination therapy, Prost et al. have demonstrated the substantial potential for drug repositioning in CM L research. Their results follow a recent report ${ }^{5}$ in which axitinib, a TKI approved for the treatment of drugresistant renal cell cancer, was repositioned for tackling TKI resistance in CM L. D rugs that are al ready approved for other purposes can shorten the drug-development pathway by 5-10 years and reduce risks and costs.

A Ithough drug repositioning can be somewhat serendipitous, Prost and colleagues had a tangible rationale that PPA $\mathrm{R}^{3}$ activators, such as pioglitazone, warranted investigation in CML, based on observed activity against a cell-line model of the disease [ok?]. A round 30\% of newly approved drugs are al ready repositioned, and such hypothesis-driven repositioning strategies are likely to become more commonplace in cancer drug discovery. A nd this figure is set to rise further as our understanding of cellular pathways and processes increases and we include innovative computational approaches to facilitate disease-, drug- and treatmentoriented drug repositioning. It is clear that repositioning will increasingly help the fasttracking of drugs into the clinic. As demonstrated by Prost and colleagues, this could soon signal the beginning of the end for stem cell quiescence in $C M L$ and other cancers.

Tessa H olyoake and David V etrie are in the Institute of Cancer Sciences, U niversity of Glasgow, Glasgow G 12 0ZD \& G61 1QH, UK.

e-mails: tessa.holyoake@glasgow.ac.uk; david.vetrie@glasgow.ac.uk

Figure 1 | Targeting leukaemic stem cells in chronic myeloid leukaemia. Prost et al. ${ }^{1}$ describe a molecular pathway, involving the receptor PPA R $\gamma$, the transcription factors STAT 5 and HIF $2 \alpha$ and the regulatory protein CITED2, that induces leukaemic stem cells 
(LSC) to enter a dormant (quiescent) state. They also show that the drug pioglitazone, which activates PPA R $\gamma$ and is approved for treating diabetes, can kill these cells when used in conjunction with tyrosine kinase inhibitors (TKIs), which are the standard therapy against active (cycling) leukaemic cells. Several additional drugs used to treat other diseases, axitinib, arsenic trioxide and hydroxychloroquine, have al so been repositioned for use in treating chronic myeloid leukaemia, but demonstrate al ternative mechanisms of action.

1. Prost Nature (2015).

2. Daley $G Q, V$ an Etten RA, B altimore D. Induction of chronic myelogenous leukemia in mice by the P210bcr/abl gene of the Philadel phia chromosome. Science. 1990 Feb $16 ; 247(4944): 824-30$.

3. Ito K, B ernardi R, M orotti A, M atsuoka S, Saglio G, Ikeda Y, Rosenblatt J , A vigan DE, Teruya-F eldstein J, Pandolfi PP. PM L targeting eradicates quiescent leukaemiainitiating cells. Nature. 2008 J un 19;453(7198):1072-8.

4. Bellodi C, Lidonnici M R, Hamilton A, Helgason GV, Soliera A R, Ronchetti M, Galavotti S, Y oung K W, Selmi T, Y acobi R, V an Etten RA, Donato N, Hunter A, Dinsdale D, Tirrò E, Vigneri P, Nicotera P, Dyer M J, Holyoake T, Salomoni P, Calabretta B. Targeting autophagy potentiates tyrosine kinase inhibitor-induced cell death in Philadel phia chromosome-positive cells, including primary CM L stem cells. J Clin Invest. 2009 M ay;119(5):1109-23.

5. Pemovska $T$, J ohnson $E, K$ ontro $M$, Repasky $G A$, Chen J, W ells $P, C$ ronin $C N$, M cTigue M, Kallioniemi O, Porkka K, M urray BW, W ennerberg K. A xitinib effectively inhibits BCR-ABL 1 (T315I) with distinct binding conformation. N ature. 2015; 519: 102-105. 
